\title{
Oilfield GIS Service Based on Mobile Platform
}

\author{
Lingling Guo ${ }^{1}$, Man Yuan ${ }^{1}$, Shaobin $\mathrm{Hu}^{2}$ \\ ${ }^{1}$ School of Computer \& Information Technology, Daqing Petroleum Institute, Daqing, China \\ ${ }^{2}$ Key Laboratory of Educational Ministry for Improving Oil and Gas Recovery, Petroleum Engineering Department, \\ Daqing Petroleum Institute, Daqing, China \\ Email: rainmanqing@yahoo.com.cn,hsbdqpi@yahoo.com.cn \\ Received January 21, 2010; revised February 27, 2010; accepted March 22, 2010
}

\begin{abstract}
With the development of mobile technology, especially the development of $3 \mathrm{G}$ and mobile IP, the computational capacity of handsets is becoming more and more powerful, which provides a new method to solve the difficulties encountered in real time GIS accessing caused by the characteristics of mobility and remoteness of fieldwork in oilfield. On the basis of studying in-depth on the technologies of J2ME platform, Mobile SVG and mobile data transfer, etc., and in accordance with the actual situation of oilfield, the design framework of oilfield mobile GIS service is put forward and the schemes of key technologies are given in this paper, which establishes the technical foundation for the construction of "Digital Oilfield".
\end{abstract}

Keywords: J2ME, Mobile Platform, GIS Service, Digital Oilfield

\section{Introduction}

Oilfields, especially the peripheral oilfields, are generally located in remote areas and the workplaces scatter widely and span large regions. The field operations, such as geophysical prospecting, drilling, logging, well testing have the characteristic of strong mobility. In this case, it is difficult to build wired network. But on the other hand, it is needed to access and control the oilfield GIS information real-timely by the "Digital Oilfield". In such a case, oilfields proposed higher requirements on the WWW-based GIS platform. In recent years, with the development of mobile communication and mobile computing technologies, it is possible for the traditional GIS to develop into mobile GIS [1-3]. The mobile GIS with handsets as the terminal is becoming a research hotspot in GIS domain. Value added service of mobile GIS in oilfield is emerging as the times require, it is meaningful to carry out the research on mobile platform-based oilfield GIS service.

Mobile GIS services are based on the technologies of Mobile SVG, J2ME platform, etc. SVG, the abbreviation of scalable vector graphics, is the formal recommendation standard of internet (W3C), and a language which uses XML to describe the two-dimensional diagrams [4]. In order to meet the requirements in wireless domain, the SVG workgroup of W3C established the specific standard Mobile SVG for the mobile application domain. For mobile developer, vector graphic has the advantages of small space usage and nice flexibility, so it is widely used in the mobile network. An important application of Mobile SVG is the location-based services, an important part of which is the handset-based map service, the product of the combination of the technologies of spatial information, mobile communication, mobile computing and so on. As a new emerging service, it has the characteristics of mobility, diversity of handsets, thin client/fat server, and limited bandwidth. J2ME is the abbreviation of Java 2 Micro Edition released by SUN, is a development platform aiming at the embedded consumption electronic products, and is a whole collection of technology and specification [5]. The discussion of this contribution is how to realize oilfield GIS service on the basis of Mobile SVG specification and J2ME platform.

Compared with the services provided by sitemap, such as the well-known Google Map, oilfield GIS service is committed to different objectives and the user groups are not the same. Microsoft, Google and Yahoo are consumer-centered to a large extent. They take mapping and geo-spatial visualization as an aspect of the search technology, trying to achieve the visualization and interrelation of global base map. While the oilfield GIS service is oilfield oriented. By using the professional data of oilfield, oilfield GIS services create the contents that can be visualized on the mobile terminal, supporting the professional and technical personnels to carry out various analyzing and decision making. 


\section{Overall Design of Mobile Platform-Based Oilfield GIS Service}

The prototype of the mobile platform-based oilfield GIS service applies $\mathrm{C} / \mathrm{S}$ structure. It is divided into three layers: data layer, middle layer and presentation layer, the hierarchical structure is showed in Figure 1.

Presentation layer: This layer implements the graphics user interface, the data representation (map display) and the simple operations on map (map zoom in or out, etc.) for various kinds of handset equipments. Because of the limited process and memory capability, most of the computing work must be implemented at the server end, while the handset can only implement the visualization of spatial data and the communication between the user and server. Due to the limited bandwidth, the SVG file transferred by the server to the handset can only contain graphic data. When map service is required by user, it sends HTTP request to the server, the server responses the user request and sends the SVG-formatted spatial data to the client, and then the client handset parses and generates the graphic map [6]. If the user needs other information query or data processing, communication with the server is required again.

Middle layer: Middle layer is made up by web server. It requests data from the data layer in accordance with the user demand, implements necessary information process and communicates with the client end. Web server acepts the service request sent by the client based on HTTP protocol, implements the data query in the back-end spatial database, then sends the query results back to the client after compression.

Data layer: It is the spatial database on the server end. It stores various geographic data needed by the mobile platform-based oilfield GIS services. After development for scores of years, oilfields have accumulated mass of data, including raw data in exploration and exploitation, results of processing and interpretation and information of surface engineering, etc., which are the basis of the spatial database. These data are classified into three sorts: basic geographic information, metadata and oilfield professional data (including gathering and transportation system for crude and gas, water injection system, water supply system, drainage system, road system, power supply system, corrosion protection and thermal insulation, communica- tion system, exploration and exploitation related mining right, reservoir boundary, basin and formation boundary, ground, fire fighting, sanitation and so on). The structure diagram of oilfield spatial attribute database is shown in Figure 2.

\section{Key Technologies}

The mobile platform-based oilfield GIS service prototype is made up by two parts: client end handset application program and Web server program. The handset application program mainly implements the interactive graphic user interface, SVG file parsing and the map display and operations (map translation, zoom in or out, etc.). The functions of Web server are mainly to accept the handset's HTTP service request, implement the data query, generate and compress the SVG map file, then send the map data file back to the client handset $[7,8]$. The structure of the prototype is shown in Figure 3.

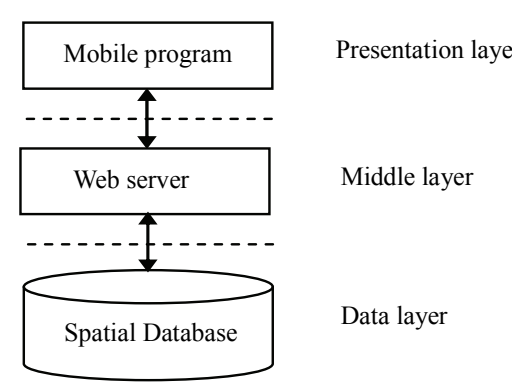

Figure 1. Layer structure of mobile platform-based oilfield GIS service.

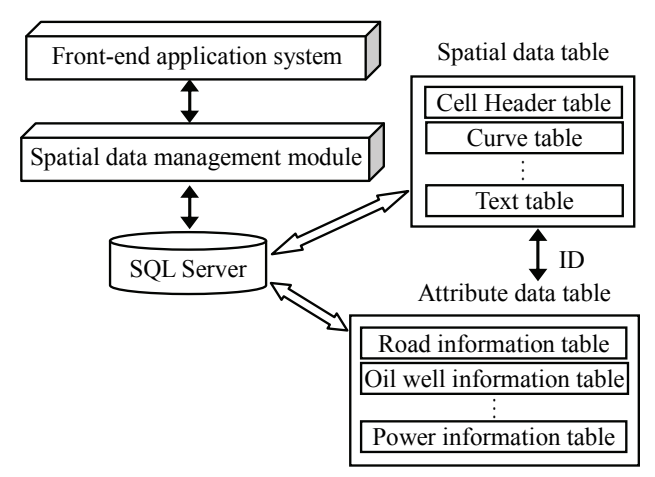

Figure 2. Structure of spatial attribute database.

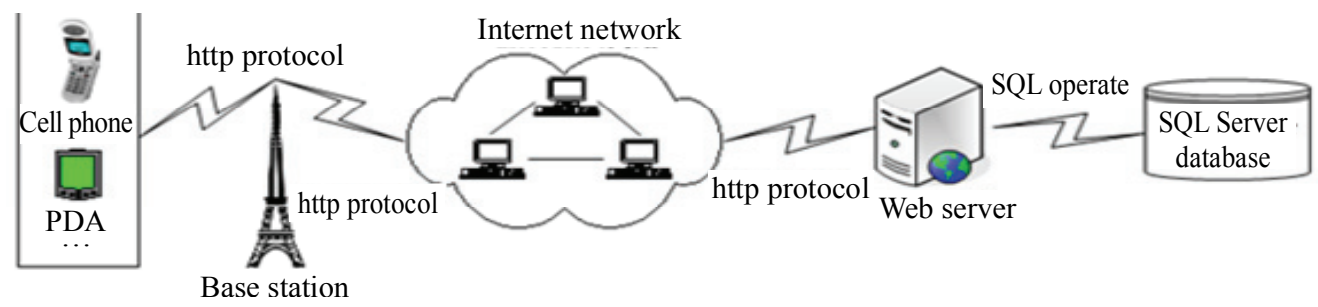

Figure 3. System architecture diagram of the prototype. 


\subsection{Generation of SVG File at the Server End}

At the server end, the file data are written to SVG file in accordance with the Mobile SVG specification, all the related geographic information needed in the SVG file are obtained by querying the spatial database, and these data are assigned to the corresponding attributes of the elements in the SVG file.

Based on the characteristics of Mobile SVG, the ground objects can be classified into point entity, linear entity and area entity according to their geometrical shape [9]. These entities are represented by corresponding graphic coding in Mobile SVG. In Mobile SVG, basic graphic elements (rectangular, circle, ellipse, line, folding line, polygon, etc.) are supported, so as the path. Various complex graphics can be plotted with path elements.

The data fields of various entity objects in the spatial database are basically the same, while the types of the spatial data of the entity objects are different. Thus, the data query should be implemented in three ways according to the entity objects. The queried contents mainly include ID, name and spatial coordinate data. As for the attributes, such as color of the entity objects, it can be acquired by querying the entity description table in the database. After the correlated data of each entity are acquired, the corresponding group and element can be generated in accordance with the Mobile SVG normative standard, and written to the SVG file as the values of the attributes of various elements, generating the demanded SVG map file.

\subsection{SVG File Compression}

Compared with other graphic formats, the SVG technology itself has the advantage of small data amount, but for the bandwidth-limited wireless network, the less the transmitted data amount, the better. As a result, compression treatment is implemented on the SVG file by using GZIP format at the server end [10].

\subsection{SVG File Parsing at the Client End}

At the mobile terminal, the SVGZ file from the server should be decompressed into SVG file. SVG file is based on XML. Presently, there are special-handset-aimed XML parsers, such as KXML [11]. Tinyline viewer is a special browser to browse the SVG file on handsets, which can be installed on handsets directly. On the other hand, one can develop the SVG file browser by himself, too. Due to the limited capacity of Tinyline viewer, a self developed browser is applied in this prototype [12].

During the process of file parsing, a third party developed SVG file parsing package, tinyline.zip, is applied. This parsing package can parse the graphic elements and their attributes in the Mobile SVG standardaccorded

SVG file. It transforms them into the J2ME graphics and displays on the handset screen.

\subsection{Map Display on Client Handset and Simple Operations}

On the client handset, simple operations on the parsed SVG file can be implemented by the user, such as map zoom in or out, translational movement, and so on.

\section{Application Example}

Through the study on the technologies of J2ME and Mobile SVG, a mobile platform-based oilfield GIS service prototype was realized by using the geographic information data of an oil production plant in Daqing oilfield. Using this prototype, one can real-timely query the surface engineering map of the plant and carry out simple operations on it, as shown in Figure 4.

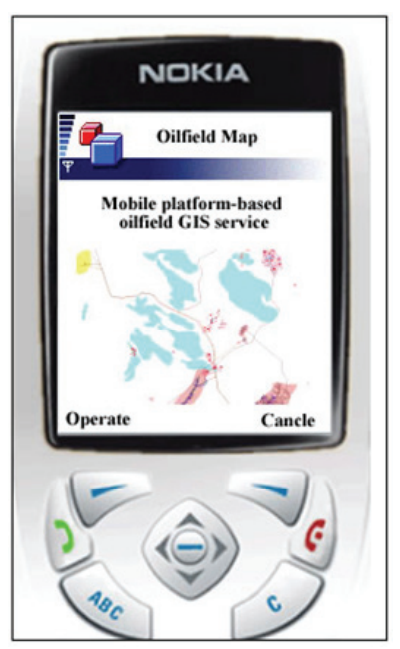

Initial interface

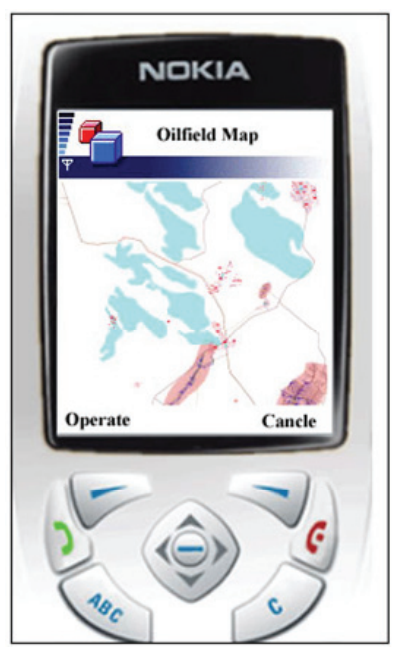

Local amplification

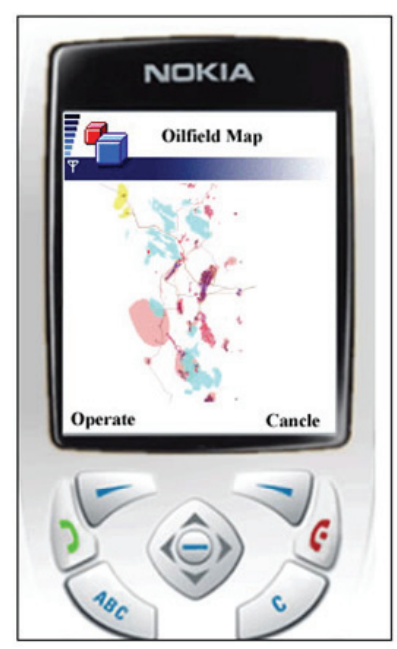

Original map

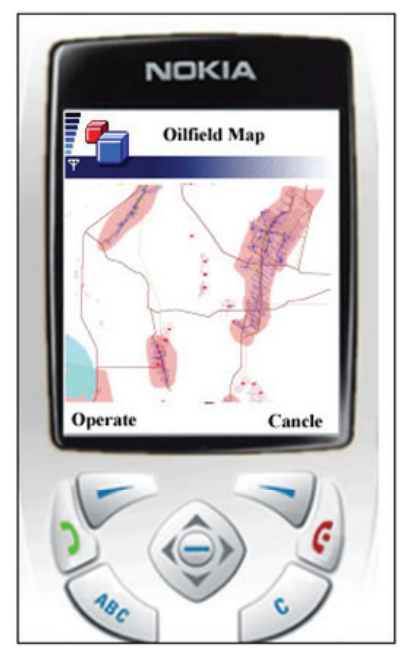

Translational movement 


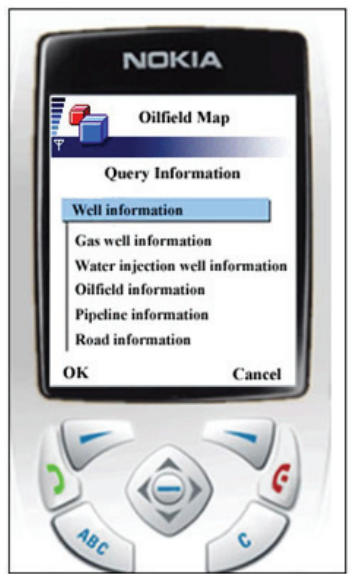

Query information

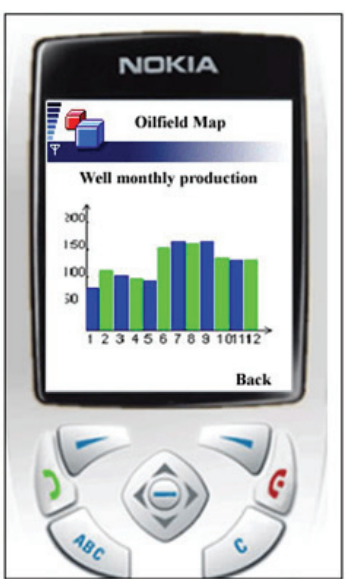

Well monthly production
Figure 4. Operation interface of surface engineering map of oilfield.

\section{Conclusions}

The design and realization of mobile platform-based oilfield GIS service prototype are introduced in this contribution. Making use of the characteristics of Mobile SVG and its advantage in the wireless graphic application, combining the J2ME development platform, the prototype organizes and codes the oilfield spatial data, then transmits the file through Web server to the client end to display. The graphics generated by using the Mobile SVG technology described in this contribution has the advantages of small size, fast access speed and easily process, therefore it is the first choice for graphic displaying at the client end of the mobile platform-based oilfield GIS service.

The study on the mobile platform-based oilfield GIS service in this contribution plays the navigation role in the "Digital Oilfield" based applications, and establishes the technical foundation for the construction of "Digital Oilfield" by using the front technology of mobile GIS.

\section{Acknowledgment}

This work is sponsored by the Supporting Program for Excellent Youth of Higher Education Institutions of Heilongjiang Province of China (1151G002); the Scientific and Technological project of Heilongjiang Province of China (2005G3674-00); the Key Scientific and Techno- logical Project of Daqing City of Chinai (SGG2005011).

\section{References}

[1] R. J. Zhang, H. Qi and W. J. Han, et al. "Design and Implement of Mobile GIS Based J2ME/Mobile SVG," Microcomputer Information, Vol. 3, No. 3, 2006, pp. 164-166.

[2] B. Z. Wu and B. Xia, "Mobile Phone GIS Based on Mobile SVG," IEEE International Geoscience and Remote Sensing Symposium, Seoul, Korea, Vol. 2, January 2005, pp. 889-892.

[3] X. Q. Zuo and Q. Q. Li, "The Deliver and Visualization of Geospatial Information in Mobile GIS," International Conference on Wireless Communications, Networking and Mobile Computing, Wuhan, September 2005, pp. 13481351.

[4] "W3C. Scalable Vector Graphics (SVG) 1.1 Specification [EB/OL]." http://www.w3.org/TR/SVG/,2003-01/2009-02

[5] "Java 2 Platform, Micro Edition (J2ME) Overview, Sun Microsystems," 10 November 2004. http://java.sun.com/ j2me/

[6] "W3C. Mobile SVG Profiles: SVG Tiny and SVG Basic [EB/OL].” http://www.w3.org/TR/2002/PR-SVGMobile20021115,2002-11/2009-02

[7] Y. G. Li, X. F. Zhou and M. Xu, "Design Mobile Commerce Security Project by Technology of J2ME and XML," Application Research of Computers, Vol. 11, 2006, pp. 105-108.

[8] "The Jakarta Site - The Apache Jakarta Tomcat, the Apahce Jakarta Project," 10 November 2004. http:// jakarta. apache. org/

[9] W. Y. Yan, "Mobile Map Service with Scalable Vector Graphics, Geoscience and Remote Sensing Symposium, 2004," IGARSS'04, Proceedings 2004 IEEE International, Anchorage, Alaska, USA, Vol. 5, January 2004, pp. 2967-2970.

[10] F. L. Zhang, X. G. He, Z. G. Qin and M. T. Zhou, "Location Management in Mobile Environment," International Conference on Communications, Circuits and Systems, January 2004, pp. 1491-1496.

[11] L. L. Guo, Z. G. Qin and M. Yuan, et al. "Mechanism of Mobile Data Transfer Based on XML," Journal of Daqing Petroleum Institute, Vol. 30, No. 5, 2006, pp. 122-124.

[12] J. H. Shin, B. J. Yi and J. J. Song, “A Development of the Mobile Computing System for Repair and Patrol of Electric Power Facilities," Fourth Annual ACIS International Conference on Computer and Information Science, January 2005, pp. 622-627. 